\title{
Celiac disease prevalence in children and adolescents with myocarditis and dilated cardiomiopathy
}

\author{
Thaysa Maria Gama Albuquerque Leão de Menezes, ${ }^{1}$ Maria Eugênia Farias Almeida Motta²
}

\begin{abstract}
Objective: To determine the prevalence of celiac disease in patients with myocarditis and dilated cardiomyopathy.

Methods: Fifty-six patients between 1 and 18 years old with dilated cardiomyopathy or myocarditis were evaluated and followed up at Instituto de Medicina Integral Professor Fernando Figueira. Patients with previous diagnosis of celiac disease were excluded. The functional classification was determined according to the American Heart Association criteria (classes I, II, III and IV). Diagnosis of myocarditis was reported in the patients' medical records. Dilated cardiomyopathy was diagnosed by echocardiogram with systolic dysfunction of one or both ventricles, ejection fraction lower than 55\%, ventricular dilatation, and left ventricular diastolic diameter bigger than $112 \%$. Patients answered a questionnaire about gastrointestinal and cardiac symptoms; next, anti-tissue transglutaminase (tTG) and anti-endomysial (EMA) antibodies were dosed. Those with positive antibody results were referred to intestinal biopsy and histological evaluation to detect celiac disease according to Marsh classification.

Results: One of the 56 children (1.8\%) had positive tTG antibody level, but negative EMA. Intestinal histological evaluation showed total villous atrophy. Approximately, $30 \%$ of patients had heart failure. Gastrointestinal symptoms and signs were frequent, especially abdominal pain $(70 \%, 39 / 56)$.

Conclusion: Celiac disease prevalence in pediatric patients with dilated cardiomyopathy or myocarditis was $1.8 \%$. It is important to investigate celiac disease in patients with these conditions to avoid the progression of such diseases and patients' clinical deterioration.
\end{abstract}

J Pediatr (Rio J). 2012;88(5):439-42: Dilated cardiomyopathy, myocarditis, prevalence, diagnosis.

\section{Introduction}

Myocarditis is an inflammatory disease and its most common cause is viral infection. ${ }^{1}$ The cause of dilated cardiomyopathy (DCM) is unknown in most cases. ${ }^{2}$ Some immunological mechanisms have been described. Underlying diseases or pathophysiological mechanisms affecting other systems may be determinants of the occurrence of heart diseases and other disorders concurrently. ${ }^{3}$

A possible underlying disease in patients with DCM/ myocarditis is celiac disease (CD). Studies in adults have shown that the prevalence of $C D$ in patients with $D C M$ ranges from 1.9 to $5.7 \% .{ }^{4-8}$ Autoimmune and inflammatory mechanisms occur in both diseases, and the alteration of intestinal permeability may facilitate the development of $\mathrm{DCM} /$ myocarditis and CD. ${ }^{3}$

Changes in the absorption of micronutrients and luminal antigens or infectious agents may promote immune and inflammatory mechanisms against antigens in the intestine and myocardium. ${ }^{9}$ Compromised intestinal permeability

1. MSc, Saúde da Criança e do Adolescente, Universidade Federal de Pernambuco (UFPE), Recife, PE, Brazil.

2. PhD, Medicina. Adjunct professor, Departamento Materno Infantil, UFPE, Recife, PE, Brazil.

No conflicts of interest declared concerning the publication of this article.

Suggested citation: de Menezes TM, Motta ME. Celiac disease prevalence in children and adolescents with myocarditis and dilated cardiomyopathy. J Pediatr (Rio J). 2012;88(5):439-42.

Manuscript submitted Jan 08 2012, accepted for publication May 302012.

http://dx.doi.org/10.2223/JPED.2219 
due to infectious or inflammatory intestinal damage can disrupt its architecture and allow endotoxin to reach the heart, changing its function and/or structure. ${ }^{10}$

Cardiologists do not suspect their patients have CD because cardiac signs and symptoms do not draw attention to this disease. Patients with heart disease have weight loss, failure to thrive, abdominal distention, and anemia. Such symptoms are common to both conditions, making it difficult to relate them to intestinal disease.

Patients with DCM/myocarditis diagnosed with $\mathrm{CD}^{4}$ showed improvement in heart volume and cardiac function with gluten-free diet. Therefore, studies investigating whether children with DCM and myocarditis also have concomitant $C D$ are needed. The present study was conducted to determine the prevalence of $C D$ in patients with $\mathrm{DCM} /$ myocarditis.

\section{Methods}

Between December 2009 and November 2010, 56 children with a clinical diagnosis of DCM/myocarditis seen at the Pediatric Cardiology Service of the Instituto de Medicina Integral Professor Fernando Figueira (IMIP) were included in our study. Myocarditis was diagnosed based on clinical criteria (tachycardia or arrhythmia, tachydyspnea, cardiomegaly, and/or transient systolic dysfunction on echocardiogram), according to admitting diagnosis, which included those who did not achieve remission (possibility of CD as a cause). Cardiac alteration of DCM was defined by the presence of at least dysfunction of the left ventricle, with ejection fraction lower than $55 \%$, ventricular dilation and left ventricular end-diastolic diameter greater than $112 \%$ on echocardiogram.

We prospectively included children older than 1 year to ensure gluten exposure. Exclusion criteria were previous diagnosis of CD. Patients answered a questionnaire about gastrointestinal and cardiovascular symptoms of DCM/ myocarditis, family history related to $C D$ and current medication. They underwent physical examination to detect signs of heart and gastrointestinal conditions.

Patients were clinically classified into heart failure (HF) functional classes according to the American Heart Association, which classifies dyspnea severity as classes I, II, III, and IV: dyspnea only with vigorous exertion, with moderate exertion, with mild exertion, and at rest, respectively.

The research project was approved by the Research Ethics Committee of IMIP, protocol number 1541-09. All participants signed an informed consent form.

Children were tested for anti-tissue transglutaminase (tTG) and anti-endomysial (EMA) Immunoglobulin A (IgA) antibodies.
Anti-tTG was measured using the Orgentec kit (Diagnostika, GmbH, Germany) according to the manufacturer's instructions. Positive results were $>10 \mathrm{U} / \mathrm{mL}$. EMA level was determined using the Immco Diagnostics kit (Genbiotech Diagnóstica Ltda., Brazil) according to the manufacturer's instructions. Positive results were > $20 \mathrm{U} / \mathrm{mL}$. Patients underwent serum IgA test (Roche Diagnostics, Germany; positivity was determined according to the manufacturer's guidelines) to rule out IgA deficiency because antibodies for detection of $C D$ are specific of the IgA class.

Intestinal biopsy was performed by means of upper digestive endoscopy when the patients had positive results for anti-tTG or EMA, for histological examination and characterization of $C D$ according to Marsh, ${ }^{11}$ considering that a CD diagnosis was established starting at Marsh II. Patients with any degree of histological changes were referred to the gastroenterology department because of higher risk of developing CD. 12

The prevalence of $C D$ was calculated based on the proportion of subjects with positive antibody test results and positive intestinal histology within the sample, with a $95 \%$ confidence interval $(95 \% \mathrm{CI})$.

\section{Results}

We investigated 56 children and adolescents, 57\% $(32 / 56)$ of them were female. The median age was 96 months (minimum $=12$ months, maximum $=225$ months). None of the patients had a diagnosis of CD before inclusion.

We found $30 \%(17 / 56)$ of patients with dyspnea, of which $23.5 \%(4 / 17)$ were in functional class IV; $29.5 \%(5 / 17)$ in functional class II; and 35.3\% (6/17) in functional class I. Two patients could not classify their dyspnea. Forty-three percent $(24 / 56)$ of patients reported prior dyspnea at the time of heart disease diagnosis. On physical examination, $51.7 \%(29 / 56)$ of subjects had mucocutaneous pallor; $44.6 \%(25 / 56)$ had heart murmur; $30.7 \%$ (17/56) had hepatomegaly; and $7 \%(4 / 56)$ had dyspnea. As for the additional tests, $57.1 \%(32 / 56)$ of patients presented with electrocardiographic change, and 59\% (33/56) had cardiomegaly on chest $X$ ray and changes in the ejection fraction.

In terms of gastrointestinal symptoms, the most frequent ones were pain $(73 \%, 41 / 56)$, abdominal distension $(59 \%, 33 / 56)$, diarrhea $(27 \%, 15 / 56)$, and pyrosis $(25 \%$, $14 / 56)$. Family members had diabetes mellitus in $30.3 \%$ of cases (17/56) and CD in 3.5\% (2/56). Hospitalization for diarrhea lasting longer than 15 days was reported by $18 \%$ of patients.

IgA deficiency was not detected. Anti-tTG serum level was positive in a female patient with intestinal biopsy showing intraepithelial lymphocytosis and total villous atrophy. 
Thus, prevalence of CD was $1.8 \%$ (95\%CI $0.04-9.5 \%$ ). EMA was negative in all patients. The patient with CD was 10 years old ( 5 years of heart disease) and had dyspnea with vigorous exertion (functional class I), without heart murmur, tachycardia, or hepatomegaly. On echocardiogram, we identified ejection fraction of $49 \%$ and enlargement of the left ventricle. This patient was using angiotensin converting enzyme inhibitor and propranolol. She reported diarrhea, abdominal distension, and pain; but she could not inform for how long she had the symptoms.

\section{Discussion}

Our study showed that children with DCM/myocarditis may have $C D$. Our patient with positive anti-tTG serum level and CD (prevalence of $1.8 \%$ ) had mild gastrointestinal symptoms (abdominal pain and distension, sporadic diarrhea), which does not draw attention to $C D$. She also had been diagnosed with heart disease 5 years earlier, with mild HF, which may not raise a suspicion of $C D$.

$\mathrm{CD}$ is frequent in patients with $\mathrm{DCM} /$ myocarditis. Studies in adults have found prevalence ranging from 1.9 to $5.7 \% .2,4,7$ There are studies showing patients with CD who developed DCM later or early changes on echocardiogram. ${ }^{9}$

Myocarditis and DCM are often found before the diagnosis of $C D$ because cardiac signs and symptoms are more prominent. The clinical presentation of CD may vary. Silent $C D$ can develop late, with extradigestive manifestations and/or mild gastrointestinal symptoms that do not raise suspicion of $C D$, especially with signs of $H F$, which diverts attention from the gastrointestinal symptoms. ${ }^{13}$

In terms of cardiac cachexia, hypoxia is mainly responsible for the change in the intestinal permeability. 3,14 Gastrointestinal symptoms draw attention to the gastrointestinal system, as these patients have protein depletion causing weakness, malnutrition, slow healing, and immunosuppression. Cardiologists should closely monitor the gastrointestinal system of HF patients because of the risk of concomitant $C D$, increased morbidity and length of hospital stay, with implications for the improvement of clinical symptoms.

Changes in the intestinal permeability may lead to $\mathrm{DCM} /$ myocarditis due to decreased absorption of important micronutrients for myocardial contraction and electrical excitability. ${ }^{15}$ Such changes also promote absorption of infectious agents that may damage the myocardium. ${ }^{3}$ Myocardial lesion may be secondary to immune response against antigens in the intestine and heart. ${ }^{3}$ Therefore, DCM/myocarditis should raise the hypothesis of a $C D$ diagnosis, since extraintestinal involvement with autoimmune mechanism may be a sing of an atypical form of CD. ${ }^{13}$ Patients with DCM/myocarditis and gastrointestinal symptoms should be periodically assessed for the possibility of gastrointestinal disease with worsening of cardiac disease.

A greater possibility of autoimmune diseases in adult patients with $C D$, namely those with delayed diagnosis has been suggested. ${ }^{4}$ There are few studies with $C D$ diagnosis in children with DCM/myocarditis. ${ }^{9}$ Elfstron et al. concluded that the CD patients' risk of developing heart disease is higher when the diagnosis is established in adulthood. ${ }^{16}$ It is possible that longer exposure to gluten coupled with autoimmune mechanisms in intestinal manifestations are responsible for the higher seroprevalence in adults. Therefore, since longer exposure to gluten seems to be necessary for the development of $C D$ and considering the possibility of association between these diseases, patients with heart disease should be evaluated for CD detection.

The few studies involving children with $C D$ have shown mild cardiac abnormalities in CD patients who consumes gluten. Polat et al. found reduction of cardiac abnormalities after starting a gluten-free diet. ${ }^{9}$

We found that children with DCM/myocarditis may have $C D$ and should be investigated. Mild gastrointestinal and cardiac symptoms should be an indication of $C D$ screening. Antibodies are highly sensitive and specific, and their identification may be a criterion for the selection of patients for intestinal histology. Gluten-free diet restores intestinal permeability, allowing adequate absorption of the drugs used in the HF treatment. Therefore, such diet may minimize the progression of the heart disease and delay indication for heart transplantation.

There are some limitations in our study. Clinical data were collected based on the patients' report on the interview, thus there may be recall bias. The sample size may seem small, but it is consistent with the number of visits at the outpatient cardiology clinic for patients with DCM/myocarditis. The etiology of DCM/myocarditis was not determined and might have interfered in the findings. There is no information on the gastroenterologic and cardiologic response of our patient after starting the gluten-free diet; therefore, we could not evaluate the association of symptoms with CD.

The present study demonstrated an association between $\mathrm{DCM} /$ myocarditis and $\mathrm{CD}$. It is important to investigate $\mathrm{CD}$ in all patients with $\mathrm{DCM} /$ myocarditis.

\section{References}

1. Calabrese F, Thiene G. Myocarditis and inflammatory cardiomyopathy: microbiological and molecular biological aspects. Cardiovasc Res. 2003;60:11-25.

2. Curione M, Barbato M, De Biase L, Viola F, Lo Russo L, Cardi E. Prevalence of coeliac disease in idiopathic dilated cardiomyopathy. Lancet. 1999;354:222-3.

3. Sandek A, Bauditz J, Swidsinski A, Buhner S, Weber-Eibel J, von Haehling $S$, et al. Altered intestinal function in patients with chronic heart failure. J Am Coll Cardiol. 2007;50:1561-9. 
4. Frustaci A, Cuoco L, Chimenti C, Pieroni M, Fioravanti G, Gentiloni $\mathrm{N}$, et al. Celiac disease associated with autoimmune myocarditis. Circulation. 2002;105:2611-8.

5. Vizzardi E, Lanzarotto F, Carabellese N, Mora A, Bertolazzi S, Benini $\mathrm{F}$, et al. Lack of association of coeliac disease with idiopathic and ischaemic dilated cardiomyopathies. Scand J Clin Lab Invest. 2008;68:692-5

6. Goel NK, McBane RD, Kamath PS. Cardiomyopathy associated with celiac disease. Mayo Clin Proc. 2005;80:674-6.

7. De Bem RS, Da Ro Sa Utiyama SR, Nisihara RM, Fortunato JA, Tondo JA, Carmes ER, et al. Celiac disease prevalence in Brazilian dilated cardiomyopathy patients. Dig Dis Sci. 2006;51:1016-9.

8. Bardella MT, Cantù P, Cesana BM, Piodi LP. Severe heart disease in coeliac sprue and inflammatory Bowel disease: a common pathogenesis? Scand J Gastroenterol. 2004;39:1304-5

9. Polat TB, Urganci N, Yalcin Y, Zeybek C, Akdeniz C, Erdem A, et al. Cardiac functions in children with coeliac disease during follow-up: insights from tissue Doppler imaging. Dig Liver Dis. 2008;40:182-7.

10. Krack A, Sharma R, Figulla HR, Anker SD. The importance of the gastrointestinal system in the pathogenesis of heart failure. Eur Heart J. 2005;26:2368-74.

11. Villanacci V, Ceppa P, Tavani E, Vindigni C, Volta U; Gruppo Italiano Patologi Apparato Digerente (GIPAD), et al. Coeliac disease: the histology report. Dig Liver Dis. 2011;43:S385-95.
12. Husby S, Koletzko S, Korponay-Szabó IR, Mearin ML, Phillips A, Shamir R, et al. European Society for Pediatric Gastroenterology, Hepatology, and Nutrition guidelines for the diagnosis of coeliac disease. J Pediatr Gastroenterol Nutr. 2012;54:136-60.

13. Fasano A. Clinical presentation of celiac disease in the pediatric population. Gastroenterology. 2005;128:S68-73.

14. Latado AL. Diet prescription in chronic heart failure: why don't we do it? Arq Bras Cardiol. 2009;93:454-5.

15. Curione M, Barbato M, Cugini P, Amato S, Da Ros S, Di Bona S. Association of cardiomyopathy and celiac disease: an almost diffuse but still less know entity. A review. Arch Med Sci. 2008;4:103-7.

16. Elfström P, Hamsten A, Montgomery SM, Ekbom A, Ludvigsson JF. Cardiomyopathy, pericarditis and myocarditis in a populationbased cohort of inpatients with coeliac disease. J Intern Med. 2007;262:545-54.

Correspondence:

Thaysa Maria Gama Albuquerque Leão de Menezes

Av. Norte, 3533/605, bloco 1 PII, Tamarineira

CEP 52051-000 - Recife, PE - Brazil

Tel.: + 55 (81) 3244.9404

E-mail: thaysamgal@gmail.com 